\title{
Bi-Layer Kinetic Inductance Detectors for W-Band
}

\author{
B. Aja ${ }^{\# 1}$, A. Gomez ${ }^{\$ 2}$, L. de la Fuente ${ }^{\#}$, A. Fernandez ${ }^{\#}$, Juan P. Pascual ${ }^{\#}$, E. Artal ${ }^{\#}$, M. C. de Ory ${ }^{*}$, M. T. \\ Magaz $\$$, D. Granados*, J. Martin-Pintado\$ \\ \#Dpt. Ing. Comunicaciones, University of Cantabria, 39005 Santander, Spain \\ ${ }^{\$}$ Centro de Astrobiología (CSIC-INTA), Torrejón de Ardoz, E-28850 Madrid, Spain \\ *IMDEA-Nanociencia, Cantoblanco, E-28049 Madrid, Spain \\ 1ajab@unican.es, ${ }^{2}$ agomez@cab.inta-csic.es
}

\begin{abstract}
A superconducting kinetic inductance detector (KID) has been fabricated and it has demonstrated absorption at W-Band. The use of a bi-layer structure based on aluminum (AI) and titanium (Ti) shows a lower superconducting critical temperature $\left(T_{c}\right)$, which allows the detection at $\mathrm{W}$-band. A design methodology is presented taking into account the KID geometry in order to maximize the absorption. A dual-polarization KID has been designed using the proposed methodology. Two prototypes of KID on Silicon substrate have been fabricated showing a good agreement between measurement and simulation results. The measurements at room temperature from 65 to $110 \mathrm{GHz}$ show the matching at the frequency band, while dark cryogenic characterization demonstrated the low frequency design.

Keywords - kinetic inductance detector, superconducting microwave devices, resonator, cryogenics.
\end{abstract}

\section{INTRODUCTION}

Over the last decades, great efforts have been done in the growth of powerful instruments to investigate the origin of the universe, in particular the Cosmic Microwave Background (CMB). The development of very sensitive detectors at $\mathrm{W}$ band is particularly interesting for CMB polarization observations since it represents the closest frequency, from ground observations, to the $70 \mathrm{GHz}$ frequency where the foreground contribution is the lowest. In the millimeter and submillimeter wave bands, different types of sensors, such as Transition Edge Sensors (TES) and Superconducting Tunnel Junctions (STJ), have shown a noise equivalent power lower than the intrinsic noise of the radiation under study [1], [2], and therefore, the further gain in sensitivity will need the increase of the number $\mathrm{n}$ of pixels, thus gaining a factor $\sqrt{n}$ in the $\mathrm{S} / \mathrm{N}$ ratio.

Among detectors based on superconducting devices, Kinetic Inductance Detectors (KIDs) provide several advantages compared with TESs, such as their intrinsic multiplexability in the frequency domain and a simpler structure [3]. Lumped-Element KIDs (LEKIDs) exploit the changes of the superconducting kinetic inductance $\left(\mathrm{L}_{\mathrm{k}}\right)$ to quantify the irradiance on each pixel. The absorbed photons change the quasiparticle density which modifies the $\mathrm{L}_{\mathrm{k}}$ of the resonator, producing a change in both resonant frequency and the quality factor of the resonator. These changes are directly proportional to the number of photons absorbed by the pixel. Thousands of LEKIDs can be read out on a single transmission line, to further increase the sensitivity.

Significant advantages have been made in developing KID [4]-[7], [3], since it was considered as a detector application for astronomy for first time in 2003 [8] specially in the submillimeter wave band. However, the KID development for the frequency range below $100 \mathrm{GHz}$ has been more recent, thanks to the use of a bi-layer combination of aluminum (Al) and titanium (Ti) [9], [10].

Although some works have shown initial developments of dual-polarization LEKIDs [7], for frequency range below $110 \mathrm{GHz}$, only single polarization detectors have been reported [10].

In this paper two LEKID prototypes are presented in Wband. Moreover, a thorough design procedure is developed considering not only the resonator itself, but also the mm-wave coupling constrains. For the first prototype, absorption measurements at room temperature are compared with simulation results, while darkness characterization was made for the second unit, made up of seven pixel array, in order to fully characterize the resonator parameters. Finally, a dualpolarization LEKID design is proposed showing the absorption efficiency for the two orthogonal polarizations in the $\mathrm{W}$ band.

\section{Kinetic InduCtANCE Detector Design}

The LEKID is a resonator of a few gigahertz composed of an inductive meander and an interdigital capacitor. The inductive meander acts as the effective optical absorbing area and is kept constant for every pixel. However, the interdigital capacitor is modified from pixel to pixel in order to tune the pixel resonant frequency $\left(\mathrm{f}_{0}\right)$, which is critical to enable a multiplexed readout scheme. In order to measure the resonant frequency, the resonator is weakly coupled to a transmission line to achieve a quality factor (Q) high enough to distinguish the shifted resonant frequency due to the absorption. The resonant frequencies of one array should be chosen to avoid overlapping of the shifted resonant frequencies after absorption.

Within Bardeen-Cooper-Schrieffer (BCS) theory, the cutoff frequency that can be absorbed by a superconductor is limited by twice the superconducting gap $\Delta$ as $2 \Delta \approx 3.52 k_{B} T_{c}$, where $k_{B}$ is the Boltzmann constant and $T_{C}$ the superconducting critical temperature. In order to push the detection to the $\mathrm{W}$ band, proximity effect is employed to diminish the Al critical temperature, and hence, the superconducting gap [9]. Following this approach, a Ti $(10 \mathrm{~nm}) / \mathrm{Al}(25 \mathrm{~nm})$ bilayer has been deposited and characterized using standard four wire resistivity measurements. The measured sheet resistances $\left(R_{s}\right)$ are $4 \mathrm{Ohm} / \mathrm{sq}$ at room temperature, and $1.27 \mathrm{Ohm} / \mathrm{sq}$ just above the critical temperature, obtaining a $T_{c}$ of $782 \pm 2 \mathrm{mK}$, as shown in Fig. 1. The obtained surface resistance is adequate to 
match the structure of the inductor at W-Band. From this results, the kinetic fraction can also be estimated using

$$
L_{k s q}=\frac{h R_{S}}{2 \pi^{2} \Delta}
$$

where $h$ is the Planck constant, obtaining $L_{k s q}=2.24 \mathrm{pH} / \mathrm{sq}$.

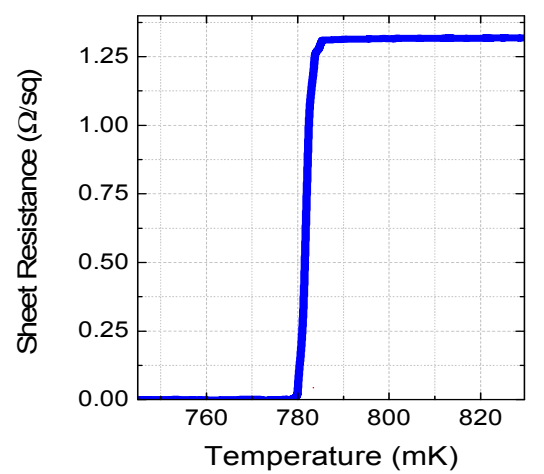

Fig. 1. Sheet resistance versus temperature of a Ti $(10 \mathrm{~nm}) / \mathrm{Al}(25 \mathrm{~nm})$ bilayer sample.

Based on these results, a developed LEKID topology takes into account both the absorption of energy at W-Band by the inductive meander as well as the quality factor of the resonator, its resonant frequency, and the obtained superconducting kinetic inductance.

The LEKID topology for the inductor consists of an Al-Ti strip grating of $3 \mu \mathrm{m}$ width and gaps of $440 \mu \mathrm{m}$, shown in Fig. 2. The $35 \mathrm{~nm}$ thickness Al-Ti film with $\mathrm{R}_{\mathrm{s}}=1.27 \mathrm{Ohm} / \mathrm{sq}$ in this strip grating has an impedance $0.840+\mathrm{j} 0.580$ at $90 \mathrm{GHz}$, normalized to the impedance of free space, $\eta_{0}=377 \mathrm{Ohm}$, when a linearly polarized incident wave has its electric field parallel to the strips [11]. The strip grating is matched to $\eta_{0}$ at $90 \mathrm{GHz}$ with a backshort at the rear side of a $0.275 \lambda$ thick Silicon ( $\mathrm{Si}$ ) substrate with dielectric constant $\varepsilon_{\mathrm{r}}=11.9$, which presents a capacitance. Each LEKID is designed to be contained in an area that is approximately $\lambda^{2}$ at the absorption frequency. Moreover, the strips are meandered together and provide a geometric inductance, $\mathrm{L}$, suitable for the desired resonance frequency.

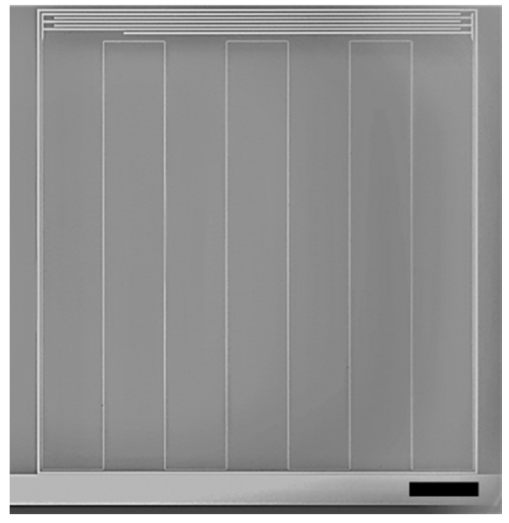

Fig. 2. SEM picture of a LEKID with size $3.2 \mathrm{~mm}$ x $3.2 \mathrm{~mm}$. Scale bar in black is $500 \mu \mathrm{m}$.
The desired resonance frequency for a first design has been set at $f_{\text {sim }}=885 \mathrm{MHz}$. The value for the geometric inductance at that frequency is $\mathrm{L}=15.7 \mathrm{nH}$, which is obtained with a quasi2D Momentum electromagnetic simulator, where the defined material is superconducting with a resistivity of the order of $10^{-8}$, without considering the kinetic inductance. Taking into account the value obtained from (1), and the geometry of the inductor, the estimated $\mathrm{L}_{\mathrm{k}}$ is $19 \mathrm{nH}$. Comparing both values, this leads to a kinetic inductance fraction of $\alpha=0.54$ with

$$
\alpha=\frac{L_{k}}{L+L_{k}}
$$

The capacitor designed is interdigital on Silicon substrate, and for the highest resonance frequency has a value of $1.035 \mathrm{pF}$, consisting of 6 fingers with $3 \mathrm{~mm}$ length, $12 \mu \mathrm{m}$ width and $15 \mu \mathrm{m}$ space between fingers.

An array of seven LEKIDs coupling them to a single 50 $\mathrm{Ohm}$ microstrip transmission line is designed and manufactured. Shield loops are added around each KID in order to diminish cross-coupling [4]. These LEKIDs are placed in a hexagonal configuration to be fed with conical horns. For frequency multiplexing, each capacitor has different value in the range from $1.035 \mathrm{pF}$ to $2.06 \mathrm{pF}$. A cylindrical waveguide with a $3.1 \mathrm{~mm}$ diameter and a vacuum gap of $70 \mu \mathrm{m}$ precedes the LEKIDs. This waveguide provides a cut-off frequency of $56.7 \mathrm{GHz}$ and confines the incident wave to the inductor area, avoiding any absorption due to the capacitor lines.

Moreover, an array of $11 \times 11$ LEKIDs in an area of $40 \mathrm{~mm} \times 40 \mathrm{~mm}$ has been fabricated in order to verify the absorption in $\mathrm{W}$-band at room temperature, which provides the frequency tuning before the optical measurements at cryogenic temperatures. The complete structure has been simulated with HFSS ANSYS 3D electromagnetic simulator at W-band. The planar-periodic structure is reduced to a single unit cell with Floquet ports, defining master and slave boundaries.

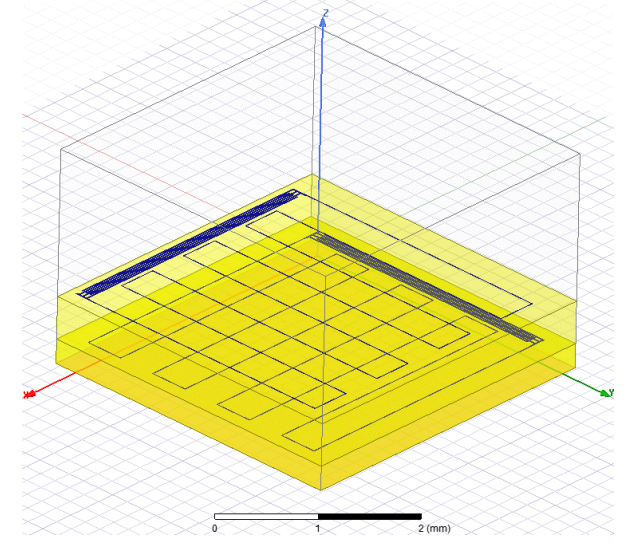

Fig. 3. Schematic of the proposed dual-polarization LEKID in W-Band. Size of the box $3.6 \mathrm{~mm} \times 3.6 \mathrm{~mm} \times 2.2 \mathrm{~mm}$ with Si substrate.

Following the BiKID approach in [12], a more advanced design of this LEKID allows to distinguish polarization between two linear polarized incident waves. Two LEKIDs are orthogonal between them and each of them is placed on both sides of a silicon wafer of thickness $483 \mu \mathrm{m}$ with electrical 
length $\lambda / 2$ at $90 \mathrm{GHz}$. A unique design with a backshort at the bottom of a $0.275 \lambda$ thick substrate of Si provides the matching of both LEKIDs at W-band. Each side of LEKIDs are coupled to a single $50 \mathrm{Ohm}$ coplanar waveguide transmission line. A schematic of the dual polarization LEKID is shown in Fig. 3. The 3D electromagnetic simulation results for the absorption in W-band of two orthogonal incident wave modes are shown in Fig. 4.

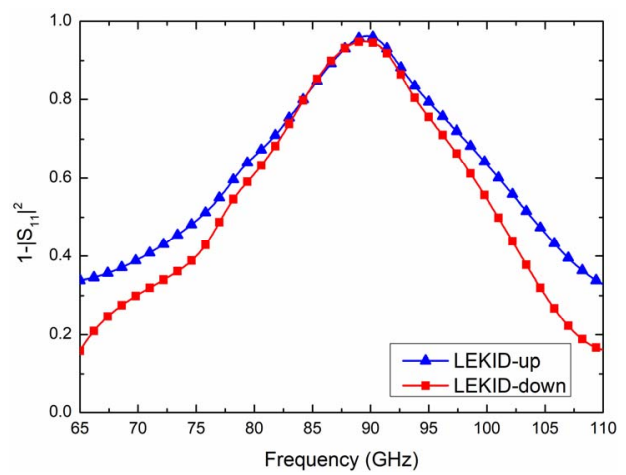

Fig. 4. Absorption of a dual-polarization LEKID in W-Band working at cryogenic temperature.

\section{KIDS NANOFABRICATION}

All the prototype devices have been fabricated using the same technological process. First, the native oxide layer of a high resistive silicon wafer $275 \mu \mathrm{m}$ thick is removed by Hydrofluoric Acid (HF) etching in order reduce two-level system noise [13]. Then, a bilayer of $\mathrm{Ti}$ and $\mathrm{Al}$ is deposited by confocal DC sputtering with base pressure better than $10^{-8}$ Torr; the confocal configuration ensures a thickness uniformity better than $3 \%$. Then, maskless laser-writer lithography on negative resist and wet etching techniques are employed in order to define the superconducting circuit. Finally, a $200 \mathrm{~nm}$ thick Al is deposited on the rear part of the Si wafer in order to act, both as ground plane and optical back short. Fig. 2 shows a Scanning Electron Microscopy picture of one fabricated LEKID with size $3.2 \mathrm{~mm} \times 3.2 \mathrm{~mm}$. Fig. 5 shows an image of the final fabricated device mounted on the chip carrier where aluminum wirebonds are used to connect the microstrip to the rest of the readout chain.

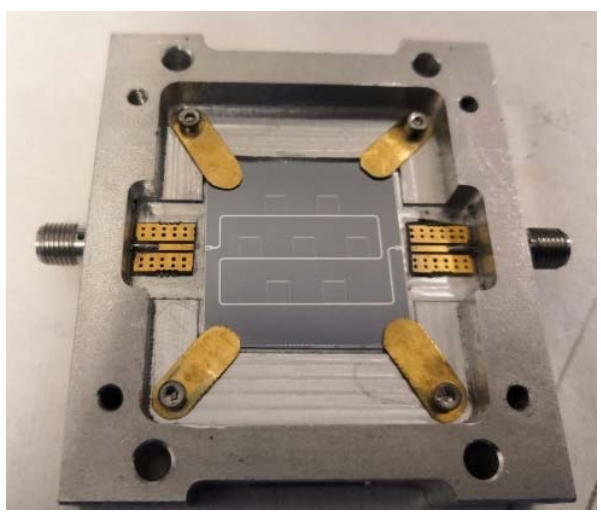

Fig. 5. Assembly of the LEKIDs on a carrier. Size of the wafer $40 \mathrm{~mm}$ x $40 \mathrm{~mm}$ with seven LEKIDs and the microstrip line.

\section{EXPERIMENTAL RESULTS}

Several measurements both room and cryogenic temperature have been carried out for the fabricated prototypes and the obtained results are compared with the simulations.

\section{A. Absorption results at ambient temperature}

A quasioptical test-bench for the LEKID characterization at W-band (75-110 GHz) is composed of feedhorns and Teflon lenses. A $4 f$ topology has been chosen with $f$ the focal length of $75 \mathrm{~mm}$, which transforms the spherical wave front radiated by the source into a plane wave front at the middle of the system with a beam waist of $16.87 \mathrm{~mm}$ [14]. Moreover, the system is formed by a PNA-X Microwave Network Analyzer Keysight N5242A, a Millimeter-Wave Controller Keysight N5261A, and two mm-wave Extension modules V10VNA2 WR-10 from OML. A photograph of the measurement set-up is shown in Fig. 6.

The absorption at ambient temperature of one array of $11 \times 11$ single-polarization LEKID was measured for linearly polarized wave. The obtained measurements together with the simulations from 65 to $110 \mathrm{GHz}$ are shown in Fig. 7. Simulations using the obtained parameters for the Ti-Al bilayers at ambient temperature $\left(\mathrm{R}_{\mathrm{s}}=4 \mathrm{Ohm} / \mathrm{sq}\right)$ confirm the experimental results, with a maximum absorption around $78 \mathrm{GHz}$.

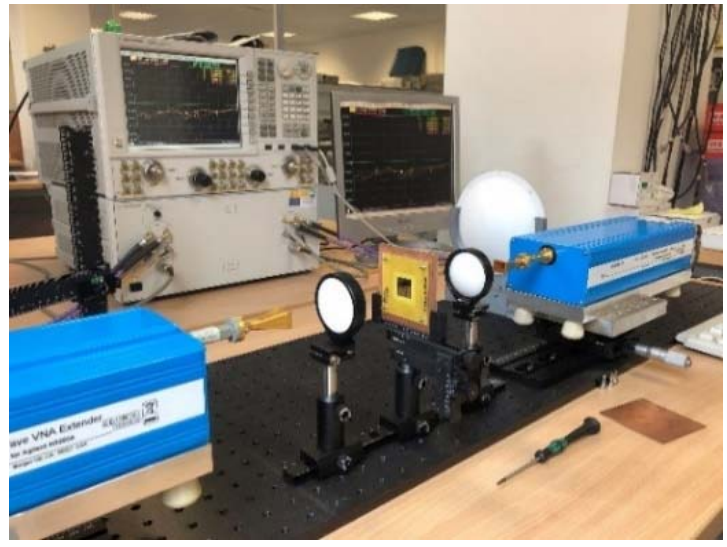

Fig. 6. Quasioptical test-bench at W-band $(75-110 \mathrm{GHz})$ for the LEKIDs characterization at ambient temperature.

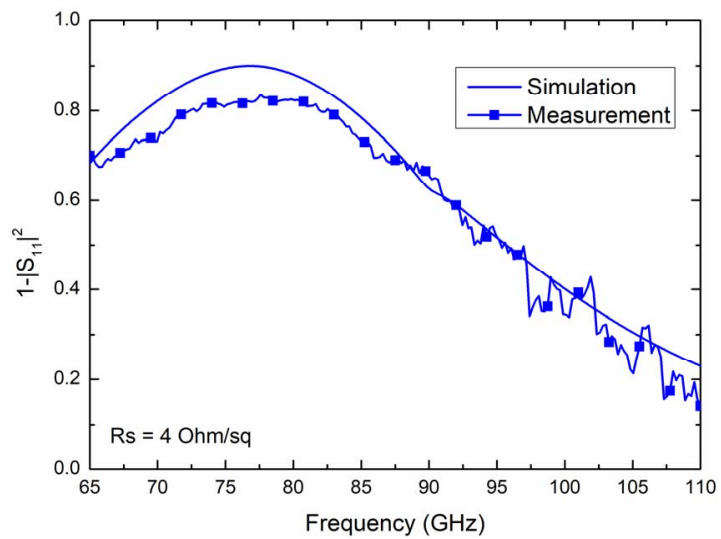

Fig. 7. Absorption measurements and simulation at W-band for the array of $11 \times 11$ single-polarization LEKID at ambient temperature. 


\section{B. Dark Cryogenic Characterization}

Cryogenic characterization was performed in a dilution refrigerator with a base temperature of $10 \mathrm{mK}$. The prototype with seven pixel array is mounted on a closed sealed metal box in order to perform a preliminary characterization in darkness conditions. Attenuators and DC blocks are used in order to diminish thermal noise and prevent thermal breaks whereas low noise amplifiers at $4 \mathrm{~K}$ and $300 \mathrm{~K}$ are used in order to amplify the output signal. A Vector Network Analyzer is employed for measuring the forward scattering parameter $\mathrm{S}_{21}$. Fig. 8 shows the obtained transmission measured at $25 \mathrm{mK}$ and readout power $-90 \mathrm{dBm}$. Each minima corresponds to a pixel and can be fitted to

$$
S_{21}=1-\frac{Q_{l}}{Q_{c}} \frac{e^{j \phi}}{1+2 j Q_{l}\left(f / f_{0}-1\right)}
$$

where $f_{0}$ is the resonance frequency, $Q_{l}$ is the loaded quality factor, $Q_{c}$ is the external quality factor and $e^{j \phi}$ accounts for the asymmetry in the resonator arisen from standing waves in the transmission line [15]. An experimental estimation of the kinetic inductance fraction can be obtained with the experimental resonance frequency, $f_{\text {exp }}$, and the simulated one $\left(f_{\operatorname{sim}}\right.$ with $\left.\mathrm{L}_{\mathrm{k}}=0\right)$ using:

$$
\alpha=1-\left(\frac{f_{\text {exp }}}{f_{\text {sim }}}\right)^{2}
$$

obtaining $\alpha=0.613$, very close to the estimated value. This result demonstrates a good sensitivity of the LEKID to an incident electromagnetic wave.

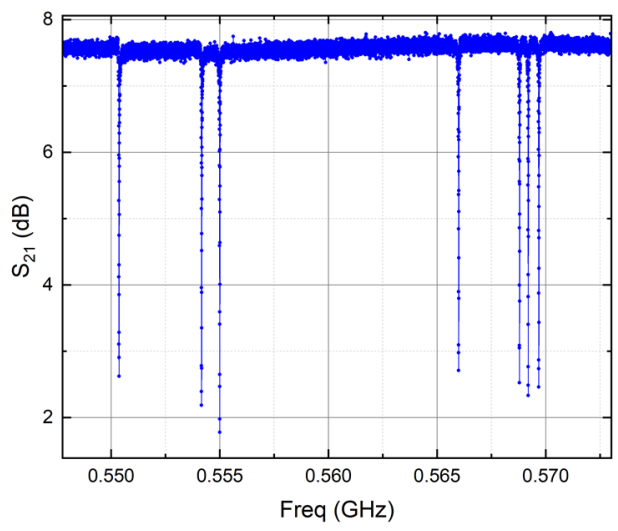

Fig. 8. Transmission measured of the array of seven LEKIDs cooled down to $25 \mathrm{mK}$ and readout power around $-90 \mathrm{dBm}$.

\section{CONCLUSION}

LEKIDs working at $\mathrm{W}$-band have been presented. Two prototypes have been fabricated and tested using an Al-Ti bilayer structure that reduces the superconducting critical temperature pushing the detection to $\mathrm{W}$-band. The room temperature characterization of one array of $11 \times 11$ singlepolarization LEKID has demonstrated a maximum absorption in W-band. On the other hand, darkness cryogenic measurements of an array of seven LEKIDs coupled to a 50 Ohm microstrip transmission line show preliminary results of the fabricated device and confirm the design methodology.

\section{ACKNOWLEDGMENT}

The authors acknowledge financial supports: Ministry of Science, Innovation and Universities Grants ESP2017-83921C2-2-R, ESP2017-86582-C4-1-R, ESP2017-86582-C4-3-R, MAT2017-85617-R, ESP2017-92706-EXP, AYA2017-92153EXP and from La Comunidad de Madrid through Grant P2018/NMT-4291 TEC2-SPACE-CM. A.G. acknowledges IJCI-2017-33991; IMDEA Nanociencia acknowledges support from the "Severo Ochoa" Programme for Centres of Excellence in R\&D (MINECO, Grant SEV-2016-0686). D.G. and A.G also acknowledge Grant DEFROST N62909-19-1-2053 from ONRGlobal.

\section{REFERENCES}

[1] M.Calvo, "Development of Kinetic Inductance Detectors for the Study of the Cosmic Microwave Background Polarization," PhD Thesis, Sapienza Universita di Roma, Italy, Oct. 2008.

[2] L. Kuzmin et al. "An Antenna Coupled Cold-Electron Bolometer for High Performance Cosmology Instruments," in Proc. 18th international Sysmposium on Space Terahertz Technology, 2007, p. 93.

[3] S. Shu et al., "Optical Response of Lumped-Element Kinetic-Inductance Detector Arrays," in IEEE Transactions on Terahertz Science and Technology, vol. 8, no. 6, pp. 605-612, Nov. 2018

[4] M. Calvo et al., "The NIKA2 Instrument, A Dual-Band Kilopixel KID Array for Millimetric Astronomy," Journal of Low Temp. Phys., vol. 184, no. 3-4, pp. 816-823, Aug. 2016.

[5] O. Noroozian, P. K. Day, B. H. Eom, H. G. Leduc and J. Zmuidzinas, "Crosstalk Reduction for Superconducting Microwave Resonator Arrays," in IEEE Transactions on Microwave Theory and Techniques, vol. 60 , no. 5, pp. 1235-1243, May 2012

[6] J. Baselmans, "Kinetic Inductance Detectors," Journal of Low Temp. Phys, vol. 167, pp. 292-304, May. 2012.

[7] McCarrick, et al, "Design and performance of dual-polarization lumpedelement kinetic inductance detectors for millimeter-wave polarimetry,", $A \& A$, vol. 610, no. A45, pp. Feb. 2018.

[8] P. K. Day, H. G. LeDuc, B. A. Mazin, A. Vayonakis and J. Zmuldzinas, "A Broadband Superconducting detector suitable for use in Large Arrays," Nature, vol. 425, pp. 817-821, Oct. 2003.

[9] A. Catalano, et al.," Bi-layer kinetic inductance detectors for space observations between 80-120 GHz," A\&A, vol. 580, pp. 1-6, Aug. 2015.

[10] A. Paiella et al, "Development of Lumped Element Kinetic Inductance Detectors for W-Band," Journal of Low Temp. Phys, vol.184, pp.97 $102,2016$.

[11] R. Ulrich, T.J. Bridges, M.A. Pollack, "Variable Metal Mesh Coupler for Fra Infrared Lasers," Applied Optics, vol. 9, no. 11, pp. 2511-2516, Nov. 1970.

[12] A. Gomez et al. "Polarization Filter for Microstrip Lumped-Element Kinetic Inductance Detectors." Journal of Low Temp.Phys., vol 193, pp 157-162, Jul 2018.

[13] J. Gao, J. Zmuidzinas, B. A. Mazin, H. G. Leduc, and P. K. Day, "Noise properties of superconducting coplanar waveguide microwave resonators," Applied Physics Letters, vol. 90, pp. 102507, Mar. 2007.

[14] P. F. Goldsmith, Quasyoptical Systems, Gaussian Beam Quasioptical Propagation and Applications, New York, IEEE Press Series on RF and Microwave Technology, 1998.

[15] S. Probst, F. B. Song, P. A. Bushev, A. V. Ustinov, and M. Weides "Efficient and robust analysis of complex scattering data under noise in microwave resonators," Review of Scientific Instruments, vol. 86, no. 2, pp. 024706, Feb. 2015. 